\title{
On Diagonal Triangle of Non Cyclic Quadrangle in Isotropic Plane
}

\section{On Diagonal Triangle of Non Cyclic Quadrangle in Isotropic Plane \\ ABSTRACT \\ Geometry of the non cyclic quadrangle in the isotropic plane was introduced in [2] and [6]. Herein, its diagonal triangle is studied and some nice properties of it are given.}

Key words: isotropic plane, non cyclic quadrangle, diagonal triangle

MSC2010: 51N25

\section{Introduction}

The isotropic plane is a real projective metric plane where metric is induced by figure consisting of an absolute point $\Omega$ and an absolute line $\omega$ incident to it. If $T=\left(x_{0}: x_{1}: x_{2}\right)$ denotes any point in the plane presented in homogeneous coordinates then usually a projective coordinate system where $\Omega=(0: 1: 0)$ and the line $\omega$ with the equation $x_{2}=0$ is chosen.

Isotropic points are the points incident with the absolute line $\omega$ and the isotropic lines are the lines passing through the absolute point $\Omega$.

Metric quantities and all the notions related to the geometry of the isotropic plane can be found in [5] and [4]. Now, we recall few facts that will be used further on wherein we assume that $x=\frac{x_{0}}{x_{2}}$ and $y=\frac{x_{1}}{x_{2}}$.

Two lines are parallel if they have the same isotropic point, and two points are parallel if they are incident with the same isotropic line.

For two non parallel points $T_{1}=\left(x_{1}, y_{1}\right)$ and $T_{2}=\left(x_{2}, y_{2}\right)$, a distance between them is defined as $d\left(T_{1}, T_{2}\right):=x_{2}-x_{1}$. In the case of parallel points $T_{1}=\left(x, y_{1}\right)$ and $T_{2}=\left(x, y_{2}\right)$, a
Dijagonalni trokut netetivnog četvreovrha $u$ izotropnoj ravnini

\section{SAŽETAK}

Geometrija netetivnog četverovrha u izotropnoj ravnini uvedena je u člancima [2] i [6]. Ovdje se proučava dijagonalni trokut i daju se neka njegova lijepa svojstva.

Ključne riječi: izotropna ravnina, netetivni četverovrh, dijagonalni trokut

span is defined by $s\left(T_{1}, T_{2}\right):=y_{2}-y_{1}$. Both quantities are directed.

Two non isotropic lines $p_{1}$ and $p_{2}$ in the isotropic plane can be given by $y=k_{i} x+l_{i}, \quad k_{i}, l_{i} \in \mathbb{R}, i=1,2$, labelled by $p_{i}=\left(k_{i}, l_{i}\right), \quad i=1,2$ in line coordinates. Therefore, the angle formed by $p_{1}$ and $p_{2}$ is defined by $\varphi=$ $\angle\left(p_{1}, p_{2}\right):=k_{2}-k_{1}$, being directed as well. Any two points $T_{1}=\left(x_{1}, y_{1}\right)$ and $T_{2}=\left(x_{2}, y_{2}\right)$ have the midpoint $M=\left(\frac{1}{2}\left(x_{1}+x_{2}\right), \frac{1}{2}\left(y_{1}+y_{2}\right)\right)$ and any two lines with the equations $y=k_{i} x+l_{i} \quad(i=1,2)$ have the bisector with the equation $y=\frac{1}{2}\left(k_{1}+k_{2}\right) x+\frac{1}{2}\left(l_{1}+l_{2}\right)$.

A triangle in the isotropic plane is called allowable if none of its sides is isotropic (see [3]).

The classification of conics in the isotropic plane can be found in [1] and [4]. To recall, the circle in the isotropic plane is the conic touching the absolute line $\omega$ at the absolute point $\Omega$. The equation of such a circle is given by $y=u x^{2}+v x+w, \quad u \neq 0, \quad u, v, w \in \mathbb{R}$.

As the principle of duality is valid in the projective plane, it is preserved in the isotropic plane as well. 


\section{Non cyclic quadrangle in isotropic plane}

We consider a complete quadrangle $A B C D$ in the isotropic plane. The points $A, B, C, D$ are the vertices of the quadrangle, and lines $A B, A C, A D, B C, B D, C D$ stand for the sides of the quadrangle $A B C D$. The pairs of sides $A B, C D ; A C, B D$ and $A D, B C$ are called the opposite sides. There is exactly one conic incident to five points $A, B, C, D$ and $\Omega$. If that conic touches the absolute line $\omega$ at the point $\Omega$, the conic is a circle and the considered quadrangle $A B C D$ is the cyclic quadrangle. In the case, when the conic intersects the line $\omega$ at the point $\Omega$ and residually at the point $\Gamma$ (different from $\Omega$ ), then the conic is a special hyperbola circumscribed to the quadrangle $A B C D$. In this case, the quadrangle $A B C D$ is called a non cyclic quadrangle. More on the geometry of such quadrangle is given in [2] and [6]. Let us denote the circumscribed special hyperbola by $\mathcal{H}$. Tangents to $\mathcal{H}$ at points $\Omega$ and $\Gamma$ are the asymptotes of this special hyperbola, $\delta$ is the isotropic one and $\gamma$ is a non-isotropic line. The intersection point $S$ of lines $\delta$ and $\gamma$ is the center of hyperbola $\mathcal{H}$. Without loss of generality affine coordinate system can be chosen in a way: $S$ is the origin, and $\gamma$ and $\delta$ stand for the coordinate axes. Although the right angle does not have any geometric sense in the isotropic plane, in the Euclidean model of the isotropic plane the coordinate system is presented as the rectangular one. Hence, due to [2] the following is valid:

Theorem 1 Any non cyclic quadrangle ABCD, by the appropriate choice of an affine coordinate system, has the vertices given with

$A=\left(a, \frac{1}{a}\right), B=\left(b, \frac{1}{b}\right), C=\left(c, \frac{1}{c}\right), D=\left(d, \frac{1}{d}\right)$,

sides of the form

$$
\begin{aligned}
& A B \ldots y=-\frac{1}{a b} x+\frac{a+b}{a b}, \\
& A C \ldots y=-\frac{1}{a c} x+\frac{a+c}{a c}, \\
& A D \ldots y=-\frac{1}{a d} x+\frac{a+d}{a d}, \\
& B C \ldots y=-\frac{1}{b c} x+\frac{b+c}{b c}, \\
& B D \ldots y=-\frac{1}{b d} x+\frac{b+d}{b d}, \\
& C D \ldots y=-\frac{1}{c d} x+\frac{c+d}{c d},
\end{aligned}
$$

and the circumscribed special hyperbola with the equation

$x y=1$.
For such quadrangle $A B C D$ from Theorem 1 it is said to be in standard position or it is a standard quadrangle. Due to Theorem 1 every non cyclic quadrangle can be represented in the standard position. So, it is sufficient to prove the properties for the standard quadrangle.

The following symmetric functions of numbers $a, b, c, d$ will be of great benefit:

$$
\begin{aligned}
& s=a+b+c+d, \\
& q=a b+a c+a d+b c+b d+c d, \\
& r=a b c+a b d+a c d+b c d, \\
& p=a b c d .
\end{aligned}
$$

The study so far ([2]) has shown several facts:

Euler circle of the triangle $A B C$ (see [3]) is given by

$\mathcal{E}_{D} \ldots a b c y=-2 x^{2}+(a+b+c) x$.

Because of symmetry on $a, b, c, d$ circles $\mathcal{E}_{A}, \mathcal{E}_{B}, \mathcal{E}_{C}$ are easy to obtain.

Due to Theorem 2 in [2] Euler circles are intersected in one point $O=(0,0)$, Euler center of the non cyclic quadrangle $A B C D$.

In [2], the forms of circumscribed circles and inscribed circles of triangles $B C D, A C D, A B D, A B C$ are obtained as well. On the example of the triangle $A B C$, its circumscribed circle is

$O_{d} \ldots a b c y=x^{2}-(a+b+c) x+b c+c a+a b$,

and its inscribed circle is given by

$\mathcal{U}_{d} \ldots 4 a b c y=x^{2}-2(a+b+c) x+(a+b+c)^{2}$.

For the triangle $A B C$, the radical axis of $O_{D}$ and $\mathcal{E}_{D}$ is an orthic of that triangle,

$\mathcal{H}_{d} \ldots 3 a b c y=-(a+b+c) x+2(b c+c a+a b)$.

Theorem 2 The median of the quadrilateral formed by the orthics of triangles $B C D, C D A, D A B, A B C$ of the standard quadrangle has the equation

$\mathcal{H} \ldots y=-\frac{s}{3 r} x+\frac{r}{3 p}$.

The principle of duality is preserved in the isotropic plane and due to [2] the medial point and the focal line of the non cyclic quadrangle are obtained as well:

Theorem 3 Bisectors of the pairs of opposite sides of the non cyclic quadrangle are passing through the one point

$N=\left(0, \frac{r}{2 p}\right)$.

The point $N$ from Theorem 3 is called a medial point of the standard quadrangle.

The common tangent of the inscribed circles $\mathcal{U}_{d}, \mathcal{U}_{c}, \mathcal{U}_{d}$ and $\mathcal{U}_{a}$ of the triangles $A B C, A B D, A C D, B C D$ is a focal line of the quadrangle $A B C D$ with equation

$\mathcal{M} \ldots y=0$. 


\section{On diagonal triangle of standard non cyclic quadrangle}

In this chapter we study diagonal triangle of the standard non cyclic quadrangle in the isotropic plane. The vertices of such diagonal triangle are intersection points of the opposite sides of quadrangle $T_{A B, C D}=A B \cap$ $C D, \quad T_{A C, B D}=A C \cap B D$ te $T_{A D, B C}=A D \cap B C$. The joint lines of these points are sides of diagonal triangle: $\mathcal{T}_{A B, C D}=T_{A C, B D} T_{A D, B C}, \mathcal{T}_{A C, B D}=T_{A B, C D} T_{A D, B C}$ and $\mathcal{T}_{A D, B C}=T_{A B, C D} T_{A C, B D}$.

The following theorems give the forms of vertices and sides of the diagonal triangle.

Theorem 4 The vertices of the diagonal triangle of the standard quadrangle $A B C D$ are given by

$$
\begin{aligned}
& T_{A B, C D}=\left(\frac{a b(c+d)-c d(a+b)}{a b-c d}, \frac{a+b-c-d}{a b-c d}\right), \\
& T_{A C, B D}=\left(\frac{a c(b+d)-b d(a+c)}{a c-b d}, \frac{a+c-b-d}{a c-b d}\right), \\
& T_{A D, B C}=\left(\frac{a d(b+c)-b c(a+d)}{a d-b c}, \frac{a+d-b-c}{a d-b c}\right) .
\end{aligned}
$$

Proof. Following equalities

$a b(a+b-c-d)=(a+b)(a b-c d)+c d(a+b)-a b(c+d)$,

$c d(a+b-c-d)=(c+d)(a b-c d)+c d(a+b)-a b(c+d)$

prove that $T_{A B, C D}$ is the intersection point of the sides $A B$ and $C D$.

Theorem 5 The diagonal triangle of the standard quadrangle $A B C D$ has the sides

$$
\begin{aligned}
& \mathcal{T}_{A B, C D} \ldots[c d(a+b)-a b(c+d)] y=(a+b-c-d) x- \\
& -2(a b-c d) \text {, } \\
& \mathcal{T}_{A C, B D} \ldots[b d(a+c)-a c(b+d)] y=(a+c-b-d) x- \\
& -2(a c-b d) \\
& \mathcal{T}_{A D, B C} \ldots[b c(a+d)-a d(b+c)] y=(a+d-b-c) x- \\
& -2(a d-b c) \text {. }
\end{aligned}
$$

Proof. According to

$$
\begin{aligned}
& (a-b+c-d)[c d(a+b)-a b(c+d)]= \\
& \quad-2(a b-c d)(a c-b d) \\
& \quad+(a+b-c-d)[a c(b+d)-b d(a+c)]
\end{aligned}
$$

and

$$
\begin{aligned}
& (a-b-c+d)[c d(a+b)-a b(c+d)]= \\
& \quad-2(a b-c d)(a d-b c) \\
& \quad+(a+b-c-d)[a d(b+c)-b c(a+d)]
\end{aligned}
$$

points $T_{A C, B D}$ and $T_{A D, B C}$ are incident to the side $\mathcal{T}_{A B, C D}$.

Theorem 6 The circle $\mathcal{K}_{D}$ circumscribed to the diagonal triangle has the equation

$$
\begin{aligned}
& \mathcal{K}_{D} \ldots[a b(c+d)-c d(a+b)][a c(b+d)-b d(a+c)] \cdot \\
& \quad \cdot[b c(a+d)-a d(b+c)] y=2(a b-c d)(a c-b d)(a d-b c) x^{2} \\
& \quad+\left\{a^{2} b^{2} c^{2}(a+b+c)+a^{2} b^{2} d^{2}(a+b+d)\right. \\
& \quad+a^{2} c^{2} d^{2}(a+c+d)+b^{2} c^{2} d^{2}(b+c+d)-2 p[a b(a+b) \\
& \quad+a c(a+c)+a d(a+d)+b c(b+c)+b d(b+d) \\
& +c d(c+d)]+3 p r]\} x
\end{aligned}
$$

The proof of the Theorem 6 is very similar to one in Theorem 5 .

Corollary 1 The circumscribed circle $\mathcal{K}_{D}$ is incident to Euler's center of the standard quadrangle.

Figure 1 presents the non cyclic quadrangle with its diagonal triangle and illustrates Corollary 1 as well.

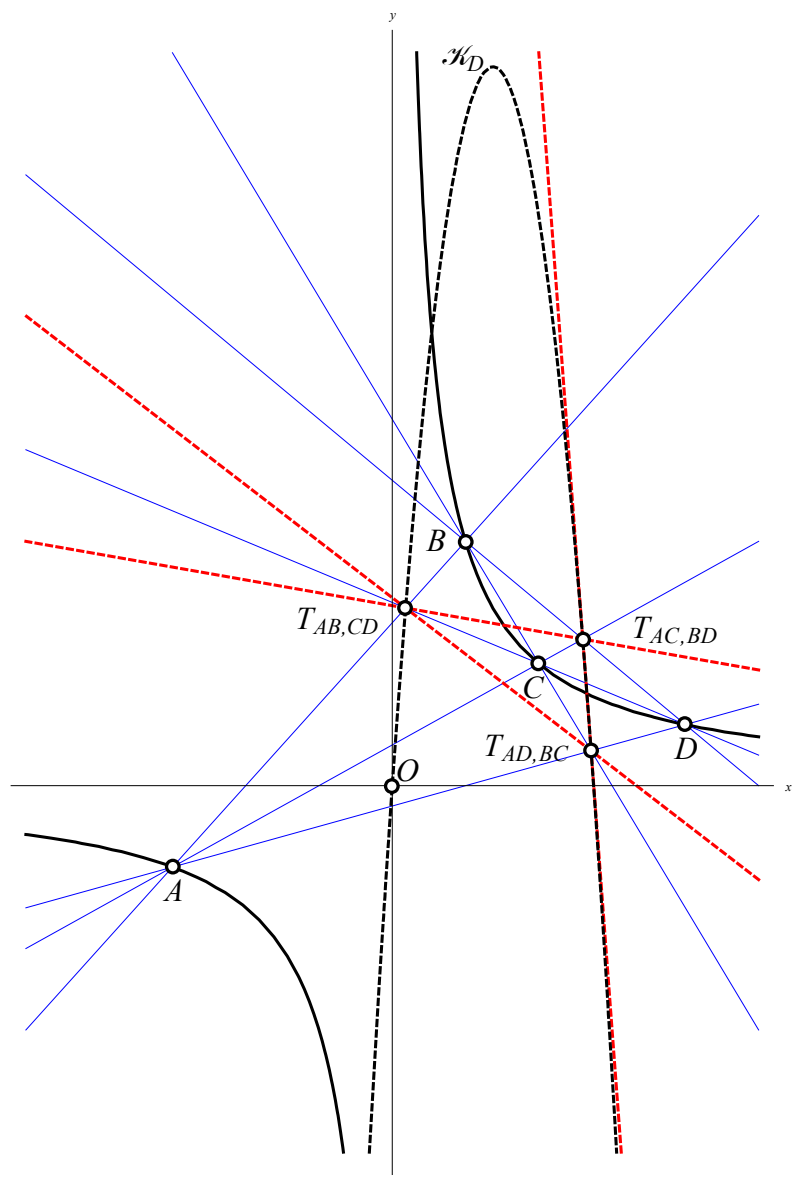

Figure 1: Non cyclic quadrangle with its diagonal triangle 
Theorem 7 If we join the points parallel to the vertices $A, B, C, D$ and incident to the sides of the diagonal triangle $\mathcal{T}_{A B, C D}, \mathcal{T}_{A C, B D}, \mathcal{T}_{A B, C D}$ to the diagonal points $T_{A B, C D}, T_{A C, B D}, T_{A D, B C}$ of the standard quadrangle $A B C D$, then they form four lines in a group $\mathcal{A}_{B}, \mathcal{B}_{A}, \mathcal{C}_{D}, \mathcal{D}_{C}$; $\mathcal{A}_{C}, \mathcal{B}_{D}, \mathcal{C}_{A}, \mathcal{D}_{B} ; \mathcal{A}_{D}, \mathcal{B}_{C}, \mathcal{C}_{B}, \mathcal{D}_{A}$. If $\mathcal{A}, \mathcal{B}, \mathcal{C}, \mathcal{D}$ are tangents to the special hyperbola circumscribed to the standard quadrangle at points $A, B, C, D$ then the quadraples of lines $\mathcal{A}, \mathcal{B}_{A}, \mathcal{C}_{A}, \mathcal{D}_{A} ; \mathcal{B}, \mathcal{A}_{B}, \mathcal{C}_{B}, \mathcal{D}_{B} ; \quad \mathcal{C}, \mathcal{A}_{C}, \mathcal{B}_{C}, \mathcal{D}_{C}$; $\mathcal{D}, \mathcal{A}_{D}, \mathcal{B}_{D}, \mathcal{C}_{D}$ are incident to four points parallel to the medial point.

Proof. The point

$$
A_{B}=\left(a, \frac{a(a+b-c-d)-2(a b-c d)}{c d(a+b)-a b(c+d)}\right) .
$$

is obviously parallel to $A$ and incident to $\mathcal{T}_{A B, C D}$ from 13 . The line $\mathcal{A}_{B}$ is of the form

$$
y=-\frac{a b-b^{2}+b c+b d-2 c d}{b(a b c+a b d-a c d-b c d)} x+\frac{2}{b}
$$

and it is joint line of the points $A_{B}$ and $T_{A B, C D}$ because of

$$
\begin{aligned}
& \frac{a(a+b-c-d)-2(a b-c d)}{c d(a+b)-a b(c+d)}= \\
& -a \frac{a b-b^{2}+b c+b d-2 c d}{b(a b c+a b d-a c d-b c d)}+\frac{2}{b}, \\
& \frac{a+b-c-d}{a b-c d}=-\frac{a b-b^{2}+b c+b d-2 c d}{b(a b-c d)}+\frac{2}{b} .
\end{aligned}
$$

Analogously, lines $\mathcal{C}_{B}$ i $\mathcal{D}_{B}$

$$
\begin{aligned}
& \mathcal{C}_{B} \ldots y=-\frac{b c-b^{2}+a b+b d-2 a d}{b(a b c+b c d-a c d-a b d)} x+\frac{2}{b}, \\
& \mathcal{D}_{B} \ldots y=-\frac{b d-b^{2}+b c+a b-2 a c}{b(b c d+a b d-a c d-a b c)} x+\frac{2}{b} .
\end{aligned}
$$

obviously pass through the point $K_{B}=\left(0, \frac{2}{b}\right)$ parallel to the medial point $N=\left(0, \frac{r}{2 p}\right)$. The line $\mathcal{B}$ given by $y=-\frac{1}{b^{2}} x+\frac{2}{b}$ is incident to the point $K_{B}$ as well.

Theorem 8 The circle that touches the focal line and the side $A B$ at the point $A$ has the common tangents to the circle $\mathcal{U}_{a}$ consisting of the focal line and the line that passes through the diagonal point $T_{A B, C D}$. There are twelwe such lines where each four of them pass through each diagonal points $T_{A B, C D}, T_{A C, B D}, T_{A D, B C}$.
Proof. The circle $\mathcal{U}_{a}$ similar to (7)

$$
4 b c d y=x^{2}-2(b+c+d) x+(b+c+d)^{2},
$$

and a circle

$4 a b^{2} y=x^{2}-2(a+2 b) x+a^{2}+4 a b+b^{2}$

have the common tangents, the focal line and the line with equation

$$
\begin{aligned}
y= & \frac{c+d-a-b}{b(a b-c d)} x+ \\
& +\frac{(a+b-c-d)\left(a b^{2}+a b c+a b d-a c d-2 b c d\right)}{b(a b-c d)^{2}} .
\end{aligned}
$$

Indeed, the equalities

$$
\begin{aligned}
& 4 b c d \frac{(a+b-c-d)\left(a b^{2}+a b c+a b d-a c d-2 b c d\right)}{b(a b-c d)^{2}}+ \\
& +4 b c d \frac{(-a-b+c+d) x}{b(a b-c d)}=x^{2}-2(b+c+d) x+(b+c+d)^{2},
\end{aligned}
$$

i.e.

$$
\left(x-\frac{a b^{2}+a b c+a b d-2 a c d-3 b c d+c^{2} d+c d^{2}}{a b-c d}\right)^{2}=0
$$

and

$$
\begin{aligned}
x^{2}- & 2(a+2 b) x+a^{2}+4 a b+4 b^{2}= \\
& =4 a b^{2}\left(\frac{(-a-b+c+d) x}{b(a b-c d)}\right. \\
& \left.+\frac{(a+b-c-d)\left(a b^{2}+a b c+a b d-a c d-2 b c d\right)}{b(a b-c d)^{2}}\right),
\end{aligned}
$$

respectively,

$$
\left(x-\frac{-a^{2} b+2 b c+2 a b d-a c d-2 b c d}{a b-c d}\right)^{2}=0
$$

show that the line $(16)$ touches the circle $\mathcal{U}_{a}$ and the circle (15) as well.

Furthermore, out of

$$
\begin{aligned}
& \frac{a+b-c-d}{a b-c d}= \\
& =\frac{(a+b-c-d)\left(a b^{2}+a b c+a b d-a c d-2 b c d\right)}{b(a b-c d)^{2}} \\
& \quad+\frac{(-a-b+c+d)[a b(c+d)-c d(a+b)]}{b(a b-c d)^{2}}
\end{aligned}
$$

it follows that the diagonal point $T_{A B, C D}$ is incident to the line (16).

It is easy to prove that the line $A B$ touches the circle $(15)$ exactly at the point $A$. 
Theorem 9 The intersection points of the bisectors of the angles of the diagonal triangle of the standard quadrangle $A B C D$ are incident to its circumscribed special hyperbola.

Proof. It is easy to show that the bisector at the vertex $T_{A B, C D}$ from 12 of the lines $\mathcal{T}_{A C, B D}$ and $\mathcal{T}_{A D, B C}$ given in (13) is of the form

$$
\begin{aligned}
\mathcal{S}_{A B, C D} \ldots y & =\left\{\frac{a+c-b-d}{2[b d(a+c)-a c(b+d)]}\right. \\
& \left.+\frac{a+d-b-c}{2[b c(a+d)-a d(b+c)]}\right\} x \\
& +\frac{a b(a+b)(c-d)^{2}-c d(c+d)(a-b)^{2}}{a^{2} b^{2}(c-d)^{2}-c^{2} d^{2}(a-b)^{2}} .
\end{aligned}
$$

Analogously, there are two more bisectors

$$
\begin{aligned}
S_{A C, B D} \ldots y & =\left\{\frac{a+b-c-d}{2[c d(a+b)-a b(c+d)]}\right. \\
& \left.+\frac{a+d-b-c}{2[b c(a+d)-a d(b+c)]}\right\} x \\
& +\frac{a c(a+c)(b-d)^{2}-b d(b+d)(a-c)^{2}}{a^{2} c^{2}(b-d)^{2}-b^{2} d^{2}(a-c)^{2}} \\
S_{A D, B C} \ldots y & =\left\{\frac{a+c-b-d}{2[b d(a+c)-a b(c+d)]}\right. \\
& \left.+\frac{a+b-c-d}{2[c d(a+b)-a b(c+d)]}\right\} x \\
& +\frac{a d(a+d)(b-c)^{2}-b c(b+c)(a-d)^{2}}{a^{2} d^{2}(b-c)^{2}-b^{2} c^{2}(a-d)^{2}} .
\end{aligned}
$$

The point of intersection $S_{A D, B C}=\mathcal{S}_{A B, C D} \cap \mathcal{S}_{A C, B D}$ is

$$
S_{A D, B C}=\left(\frac{a d(b+c)-b c(a+d)}{a d-b c}, \frac{a d-b c}{a d(b+c)-b c(a+d)}\right) .
$$

For example, the equality

$$
\begin{gathered}
\frac{a d-b c}{a d(b+c)-b c(a+d)}=\left\{\frac{a+b-c-d}{2[c d(a+b)-a b(c+d)]}+\right. \\
\left.+\frac{a+d-b-c}{2[b c(a+d)-a d(b+c)]}\right\} \frac{a d(b+c)-b c(a+d)}{a d-b c}+ \\
+\frac{a c(a+c)(b-d)^{2}-b d(b+d)(a-c)^{2}}{a^{2} c^{2}(b-d)^{2}-b^{2} d^{2}(a-c)^{2}}
\end{gathered}
$$

proves that $S_{A D, B C}$ is incident to the bisector $S_{A C, B D}$. Because of symmetry $a, b, c, d$ there are two more similar intersections

$$
\begin{aligned}
& S_{A C, B D}=\left(\frac{a c(b+d)-b d(a+c)}{a c-b d}, \frac{a c-b d}{a c(b+d)-b d(a+c)}\right), \\
& S_{A B, C D}=\left(\frac{a b(c+d)-c d(a+b)}{a b-c d}, \frac{a b-c d}{a b(c+d)-c d(a+b)}\right) .
\end{aligned}
$$

Obviously, they all lie on the special hyperbola $x y=1$.

All Theorems 7 9 have no analogous in Euclidean plane.

\section{References}

[1] J. BebAn-Brkić, M. ŠImić, V. Volenec, On Foci and Asymptotes of Conics in Isotropic Plane, Sarajevo J. Math. 3(16) (2007), 257-266.

[2] J. Beban-Brkić, M. Šimić , V. Volenec, On some properties of non-cyclic quadrangle in isotropic plane, Proceedings of 13th International Conference on Geometry and Graphics, Dresden, 2008.

[3] R. KOLAR-ŠUPer, Z. KOLAR-Begović, V. VOLENEC, J. BEBAN-BRKIĆ, Metrical relationships in standard triangle in an isotropic plane, Math. Commun. 10 (2005), 159-167.

[4] H. SACHS, Ebene isotrope Geometrie, ViewegVerlag, Braunschweig-Wiesbaden, 1987.

[5] K. Strubecker, Geometrie in einer isotropen Ebene, Math. Naturwiss. Unterr. 15 (1962-63), 297 306, 343-351, 385-394.

[6] M. Šimić, V. Volenec, J. Beban-Brkić, On umbilic axes of circles of the non-cyclic quadrangle in the isotropic plane, Math. Pannon. 21(2) (2010), 112.

\section{Marija Šimić Horvath \\ orcid.org/0000-0001-9190-5371 \\ e-mail: msimic@arhitekt.hr}

Faculty of Architecture, University of Zagreb

Kačićeva 26, HR-10000 Zagreb, Croatia

\section{Vladimir Volenec}

orcid.org/0000-0001-7418-8972

e-mail: volenec@math.hr

Faculty of Science, University of Zagreb

Bijenička cesta 30, HR-10000 Zagreb, Croatia 